\title{
Computational Mining and Genome Wide Distribution of Microsatellite in Fusarium oxysporum f. sp. lycopersici
}

\author{
Sudheer KUMAR*, Deepak MAURYA, Shalini RAI, Prem \\ Lal KASHYAP, Alok Kumar SRIVASTAVA \\ National Bureau of Agriculturally Important Microorganisms (NBAIM), Mau, Uttar Pradesh, \\ 275101, India; sudheer.nbaim@gmail.com ( ${ }^{*}$ corresponding author)
}

\begin{abstract}
Simple sequence repeat (SSR) is currently the most preferred molecular marker system owing to their highly desirable properties viz., abundance, hyper-variability, and suitability for high-throughput analysis. Hence, in present study an attempt was made to mine and analyze microsatellite dynamics in whole genome of Fusarium oxysporum $\mathrm{f}$. sp. lycopersici. The distribution pattern of different SSR motifs provides the evidence of greater accumulation of tetra-nucleotide (3837) repeats followed by tri-nucleotide (3367) repeats. Maximum frequency distribution in coding region was shown by mono-nucleotide SSR motifs (34.8\%), where as minimum frequency is observed for penta-nucleotide SSR (0.87\%). Highest relative abundance (1023 SSR/Mb) and density of SSRs (114.46 bp/Mb) were observed on chromosome 1, while least density of SSR motifs was recorded on chromosome $11(7.40 \mathrm{bp} / \mathrm{Mb})$ and 12 (7.41 bp/Mb), respectively. Maximum trinucleotide (34.24\%) motifs code for glutamic acid (GAA) while GT/CT were the most frequent repeat of dinucleotide SSRs. Most common and highly repeated SSR motifs were identified as (A)64, (T)48, (GT)24, (GAA)31, (TTTC)24, (TTTCT) 28 and (AACCAG)27. Overall, the generated information may serve as baseline information for developing SSR markers that could find applications in genomic analysis of $F$. oxysporum $\mathrm{f}$. sp. lycopersici for better understanding of evolution, diversity analysis, population genetics, race identification and acquisition of new virulence.
\end{abstract}

Keywords: amino acid, chromosome, codon, microsatellite, SSR

\section{Introduction}

Fusarium oxysporum f. sp. lycopersici, the cause of tomato crown and root rot is an important soil-borne fungus and reduce crop productivity by $10-50 \%$ (Borrero et al., 2004). The use of resistant varieties is the most economical and effective way to manage the disease. However, new races of pathogen have been emerged that overcome resistance in currently growing tomato cultivars (Mishra et al., 2010). Therefore, knowledge of the genetic variation within and among populations is an important component to understand the population biology of $F$. oxysporum f. sp. lycopersici for developing strategies to enhance the durability of resistance. Virulence tests are commonly used to detect the pathogen variations (Elias et al., 1991) and three distinct races (1,2 and 3) of F. oxysporum f. sp. lycopersici have been identified (Cai et al., 2003). However, these tests are subjected to availability of host selection pressure, tedious, inconclusive and preclude nonpathogenic strains. To circumvent these problems, DNA based molecular markers have been used in diversity analysis, virulence evaluation and genetic structure of pathogen races (Lievens et al., 2009).

Simple sequence repeat (SSR) or microsatellite markers have become a preferred choice in recent years for sev- eral uses due to their multi-allelic nature, co-dominant inheritance, high abundance, hyper variance, extensive genome coverage, reproducibility, and discriminatory power (Mahfooz et al., 2012). Except for some nuclear restriction fragment length polymorphism (RFLP) (Rosewich et al., 1999) and RAPD (random amplified polymorphic DNA) markers (Balmas et al., 2005), limited molecular markers were available for $F$. oxysporum f. sp. lycopersici genetic studies. Nevertheless, recent availability of genome sequence information of $F$. oxysporum $\mathrm{f}$. sp. lycopersici has provided the opportunity to study the genome wide distributional pattern of SSRs motifs in tomato root rot pathogen. This study describes comprehensive report on mining and analysis of microsatellite dynamics in $F$. oxysporum $f$. sp. lycopersici using bioinformatics approaches.

\section{Materials and methods}

\section{Retrieval of sequences}

The nuclear and mitochondrial genome sequences of F. oxysporum f. sp. lycopersici (strain 53 4287, race 2, VCG 0030) available in Fusarium Comparative Database of Broad Institute of MIT and Harvard, Cambridge (http:// www.broadinstitute.mit.edu/) were used for the present study. 
128

\section{Microsatellite mining}

The retrieved sequences were analyzed for repeat patterns using WebSat (SSR finder program) (Martins et al., 2009). The generated data was further used for screening of SSR containing sequences by Simple Sequence Repeat Identification Tool (SSRIT). The program was run online and the parameters were set for detection of perfect di-, tri-, tetra-, penta- and hexa-nucleotide motifs with a minimum of six repeats. The data were processed and counted with Microsoft Excel 2007.

\section{Statistical analysis}

The analysis of SSRs was done based on their types (mono- to hexa-nucleotides), number of repeats, frequency of occurrences of each SSR motif and their distribution in the sequence. The relative abundance and density were calculated by following formulas:

Relative abundance $=$ Number of SSRs $/$ Length of sequence analyzed $(\mathrm{Mb})$;

Relative density = Length of SSR (bp) / Length of sequence analyzed $(\mathrm{Mb})$.

\section{Results}

\section{Abundance and density of microsatellite}

Total genome sequence data $(59.9 \mathrm{Mb})$ of $F$. oxysporum f. sp. lycopersici was assembled into 423 scaffolds and used to explore mono-, di-, tri-, tetra-, penta- and hexa-nucleotide motifs with a repeat of $\geq 6$ times. A total 13864 SSRs were identified from whole genome data of $F$. oxysporum f. sp. lycopersici (Tab. 1). The relative abundance and density of SSRs were $231.45 \mathrm{SSR} / \mathrm{Mb}$ and $2643.73 \mathrm{bp} / \mathrm{Mb}$, respectively (Tab. 1).

The number of repeat units in di-, tri-, tetra-, pentaand hexanucleotides ranged from 10 to 46 , but the maTab. 1. Number and distribution of SSRs in whole genome of $F$. oxysporum f. sp. lycopersici

\begin{tabular}{cc}
\hline Total size covered by examined sequences $(\mathrm{Mb})$ & 59.9 \\
\hline Total number of SSR identified & 13864 \\
\hline Perfect SSR & 13608 \\
Compound SSR & 256 \\
\hline Imperfect SSR & 139 \\
\hline Total relative abundance $(\mathrm{SSR} / \mathrm{Mb})$ & 231.45 \\
\hline Total relative density $(\mathrm{bp} / \mathrm{Mb})$ & 2643.73 \\
\hline
\end{tabular}

jority of SSRs (78.25\%) had 6 to 31 repeat units. Tetra(3837) and tri-nucleotide (3367) repeats were the most abundant repeats in the genome accounting $27.67 \%$ and $24.28 \%$ SSRs followed by penta- $(18.09 \%)$ and tri-nucleotides (17.10\%) repeats (Tab. 2). Maximum frequency distribution in coding region was shown by tri-nucleotide SSR motifs i.e. $34.8 \%$ in contig, whereas minimum frequency are observed for penta-nucleotide SSR in contig $(0.87 \%)$. Mono-, di-, tetra- and penta-nucleotide repeats were the least frequent accounting less than $10 \%$ of SSRs in the coding region of genome. Among the mononucleotides, polyT (64) was the longest SSR motif (Tab. 2). The number of repeat units ranged from 22 to 64 among mono-nucleotides, but majority of repeats had 22 repeat units. Some of the highly repeated sequences identified were (A)64, (T)48, (GT)24, (GAA)31, (TTTC)24, (TTTCT)28 and (AACCAG)27 (Tab. 2).

\section{Microsatellite distribution on chromosome}

Chromosome 1 possessed highest number of SSRs (7008) and chromosome 13 had the least number of SSRs (119) (Tab. 3). Four hundred and twenty three SSRs were identified in the contigs not mapped to any chromosome. Tetra-nucleotides repeats were the most abundant (3837) repeats in all the chromosomes accounting $27.67 \%$ of SSRs. Maximum relative abundance (1023 SSR/Mb) and density of SSRs (114.46 bp/Mb) was occurred on chromosome 1 and followed by chromosome 2 (148 SSR/ $\mathrm{Mb}, 18.1 \mathrm{bp} / \mathrm{Mb}$ ), chromosome 7 (147 SSR/Mb, 16.55 $\mathrm{bp} / \mathrm{Mb})$, chromosome 8 (146 SSR/Mb, $17.17 \mathrm{bp} / \mathrm{Mb})$, chromosome $15(145 \mathrm{SSR} / \mathrm{Mb}, 18.6 \mathrm{bp} / \mathrm{Mb})$ and $14(144$ SSR/Mb, $21.6 \mathrm{bp} / \mathrm{Mb}$ ), respectively (Tab. 3). Least relative abundance of repeat motifs were occurred on chromosome 12 (68 SSR/Mb) and 13 (68 SSR/Mb), while least density was observed on chromosome $11(7.40 \mathrm{bp} / \mathrm{Mb})$ and $12(7.41 \mathrm{bp} / \mathrm{Mb})$, respectively (Tab. 3).

\section{Frequency of microsatellite classes}

SSRs were categorized into three groups based on length of SSR tracts (Fig. 1). Class I, II and III SSRs contain perfect repeats $\geq 10,10-20$ and $<20$ nucleotides in length, respectively. Out of 13180 SSRs, 269 repeats (2.04\%) were categorized as Class I SSRs. About 9.87\% and $88.08 \%$ SSRs in F. oxysporum f. sp. lycopersici genome were classified in Class II and Class III, respectively.

Tab. 2. Distribution of SSR motifs in coding and non-coding regions for F. oxysporum f. sp. lycopersici

\begin{tabular}{ccccccc}
\hline \multirow{2}{*}{ Motif length } & \multirow{2}{*}{ Total } & \multicolumn{2}{c}{ Coding region } & \multicolumn{2}{c}{ Non-coding region } & \\
\cline { 3 - 6 } & & Number & $\%$ & Number & $\%$ & \multirow{2}{*}{ Longest SSR motifs * } \\
\hline Mono & 2371 & 106 & 4.5 & 2265 & 95.5 & A (48), T (64), G (22), C (22) \\
Di & 522 & 17 & 3.25 & 505 & 96.75 & AT (19), TG (19), GT (24), CT (20) \\
Tri & 3367 & 1172 & 34.8 & 2195 & 65.2 & CAG (10), TTC (17), GAA (31), ATT (25) \\
Tetra & 3837 & 209 & 5.4 & 3608 & 94.6 & CTTG (10), TTTC (24), GTAG (10), AAAG (19) \\
Penta & 2509 & 22 & 0.87 & 2487 & 99.13 & CTCTT (11), TTTCT (28), GAGAA (6), AGCAT (11) \\
Hexa & 1258 & 248 & 19.7 & 1010 & 80.3 & CTAACC (20), TGGCTC (12), GGGTA (16), AACCAG (27) \\
\hline
\end{tabular}

Number in parenthesis represents the number of repeats in longest SSR 
Tab. 3. Distribution of SSRs in different chromosomes identified from public available whole genome database of $F$. oxysporum $\mathrm{f}$. sp. lycopersic

\begin{tabular}{cccccccccccc}
\hline Chromosome & Mono & Di & Tri & Tetra & Penta & Hexa & Size $(\mathrm{Mb})$ & SSR $(\mathrm{Mb})$ & bp $(\mathrm{Mb})$ & SSR \\
\hline 1 & 1102 & 296 & 1777 & 1802 & 1358 & 673 & 6.85 & 1023 & 114.46 & 7008 \\
2 & 140 & 35 & 216 & 176 & 176 & 88 & 5.58 & 148 & 18.1 & 831 \\
3 & 204 & 19 & 128 & 202 & 96 & 40 & 5.63 & 122 & 13.23 & 689 \\
4 & 110 & 21 & 155 & 148 & 86 & 52 & 5.21 & 109 & 12.86 & 572 \\
5 & 91 & 25 & 160 & 231 & 119 & 72 & 4.91 & 142 & 17.07 & 698 \\
6 & 128 & 12 & 96 & 158 & 85 & 34 & 4.59 & 111 & 11.95 & 513 \\
7 & 99 & 25 & 153 & 180 & 126 & 60 & 4.35 & 147 & 16.55 & 643 \\
8 & 92 & 15 & 139 & 165 & 105 & 67 & 3.98 & 146 & 17.17 & 583 \\
9 & 65 & 18 & 110 & 122 & 89 & 38 & 3.3 & 133 & 17.22 & 442 \\
10 & 75 & 10 & 97 & 128 & 63 & 36 & 2.9 & 141 & 21.59 & 409 \\
11 & 30 & 4 & 56 & 59 & 19 & 9 & 2.34 & 75 & 7.402 & 177 \\
12 & 26 & 10 & 43 & 46 & 19 & 8 & 2.23 & 68 & 7.41 & 152 \\
13 & 10 & 2 & 44 & 36 & 14 & 13 & 1.75 & 68 & 8.86 & 119 \\
14 & 36 & 10 & 45 & 99 & 45 & 17 & 1.65 & 144 & 21.6 & 252 \\
15 & 63 & 8 & 75 & 147 & 41 & 19 & 2.43 & 145 & 18.6 & 353 \\
Scaffold & 100 & 12 & 73 & 138 & 68 & 32 & 3.75 & 112 & 7.592 & 423 \\
Total & 2371 & 522 & 3367 & 3837 & 2509 & 1258 & 59.1 & 234 & 2643.73 & 13864 \\
\hline
\end{tabular}

\section{Codon repetition and amino acid distribution}

Tri-nucleotide SSRs are triplet codon that code for a particular amino acid. It was observed that out of all triplet codons of contig sequences, GAA (encoding glutamic acid) repetitions are predominant (34.24\%) and followed by ATT (encoding isoleucine) and TTC (encoding phenylalanine) (Tab. 4). Analysis of all coded amino acid in contigs sequences demonstrated that the serine (391) and leucine (386) had the highest occurrence followed by arginine (248) (Tab. 4). Tryptophane (57), methionine (58) and asparagine (59) were the least occurred amino acids in the ESTs of $F$. oxysporum f. sp. lycopersici (Tab. 4).

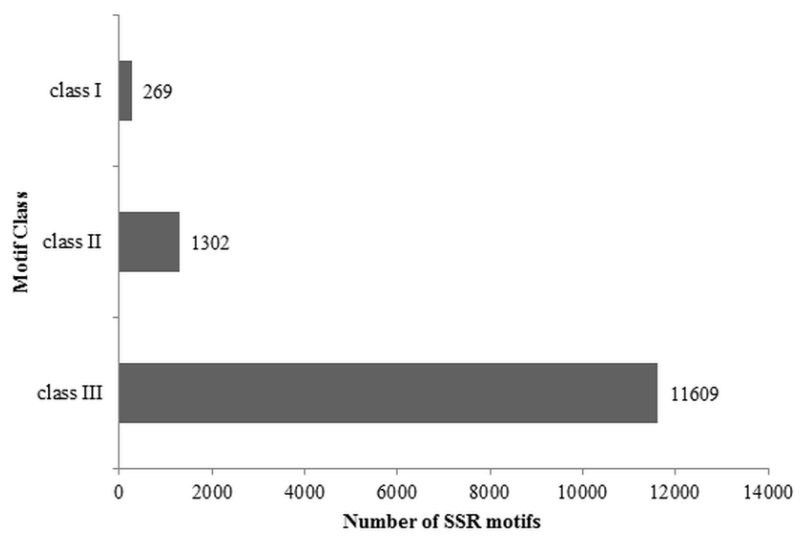

Fig. 1. Frequency of Class I, II and III SSRs in F. oxysporum f. sp. lycopersici genome

\section{Discussion}

Simple sequence repeat (SSR) is currently the most preferred molecular marker system owing to their highly desirable properties viz., abundance, hyper-variability, and suitability for high-throughput analysis. Several studies have shown the importance of using microsatellites to understand epidemiological processes in plant pathogenic fungi (Breuillin et al., 2006; Lievens et al., 2009). Their codominance, high polymorphism, and ease of scoring allow inferences of population genetic parameters such as gene flow, effective population size, or reproductive system, to be made with high accuracy (Mahfooz et al., 2012). Most importantly, microsatellite sequences obtained through in silico mining have more or less the same utility and potential comparative with those derived from a genomic library. However, the negligible cost of in silico mining and high abundance of microsatellites in different sequence resources make this approach extremely attractive for the generation of microsatellite markers. Therefore, in present study, computational approaches were employed to mine and analyze genome wide distribution patter of microsatellite in Fusarium oxysporum f. sp. lycopersici.

The present study clearly demonstrates that the distribution of microsatellites in the genome is non-random, presumably because of their effects on chromatin organization, regulation of gene activity, recombination, DNA replication, cell cycle, mismatch repair system etc. ( $\mathrm{Li}$ et al., 2002, 2004). Coding regions are mostly dominated by tri- and hexa- repeats, whereas di-, teta-, and hexa- nucleotide repeats are often found in non-coding regions. Similar, distribution pattern of SSR motifs and predominance of tri- and hexa- motifs in the coding region was reported by Mahfooz et al. (2012). These tri- and hexa- SSR motifs in the coding regions are translated into amino acids repeats, which possibly contribute to the biological function of the protein (Kim et al., 2008). Di-nucleotide motifs are often found in the exonic region of $F$. oxysporum (Mahfooz et al., 2012), however, (GT)n repeats were also com- 
Tab. 4. Different types of amino acid and their distribution in F. oxysporum f. sp. lycopersici genome

\begin{tabular}{ccc}
\hline Total Number & Nucleotide sequence \\
\hline Alanine & 154 & GCT/GCC/GCA/GCG \\
\hline Arginine & 248 & AGA/AGA/AGG/CGT/CGC/CGG/CGA \\
\hline Asparagine & 59 & AAC/AAT \\
\hline Aspartic Acid & 140 & GAT/GAC \\
\hline Cysteine & 129 & TGT/TGC \\
\hline Glutamic Acid & 214 & GAG/GAA \\
\hline Glutamine & 145 & CAA/CAG \\
\hline Glycine & 121 & GGT/GGC/GGA \\
\hline Histadine & 113 & CAC/CAT \\
\hline Isoleucine & 146 & ATA/ATC/ATT \\
Leucine & 386 & CTT/CTG/CTA/CTC/TTA/TTG \\
\hline Lysine & 88 & AAG \\
Methionine & 58 & ATG \\
\hline Phenylalanine & 97 & TTC \\
\hline Proline & 117 & CCT/CCG/CCA \\
Serine & 391 & TAA/TAG/TGA \\
\hline Stop Codon & 139 & TCA/TCC/TCT/TCG/AGT/AGC \\
\hline Threonine & 100 & ACT/ACA/ACG/ACC \\
\hline Tryptophan & 57 & TGG \\
Tyrosine & 90 & TAT/TAC \\
Valine & 160 & GTC/GTA/GTG/GTT \\
\hline
\end{tabular}

mon in the F. oxysporum f. sp. lycopersici. Stallings et al. (1991) reported that $(\mathrm{GT}) \mathrm{n}$ repeat is able to enhance the gene activity from a distance independent of its orientation. However, more effective transcription enhancement results from GT repeats being closer to promoter region.

The frequency distribution by repeat types shows major differences in various genomic regions (Tóth et al., 2000). Tri-nucleotide repeats have been found to be common feature in EST-derived SSRs in present study. High frequency of these repeats in coding regions could be due to mutation and selection pressure for specific amino acids (Morgante et al., 2002). The abundance of trinucleotide repeats EST-SSR is likely due to suppression of other kind of repeats in the coding region, which reduces the frameshift mutations in the coding regions (Metzgar et al., 2000). GAA repeats are very abundant in $F$. oxysporum $\mathrm{f}$. sp. lycopersici coding regions, and found very rare in the exons of F. graminearum coding region exons (Singh et al., $2011 \mathrm{a}$ ), and CTT repeat motif, relatively abundant in $F$. graminearum exons, are uncommon in F. oxysporum f. sp. lycopersici. These differences could be due to differences in the slippage process, or they may reflect the low GC content of the genome (Richard and Dujon, 1997). The chromosomal location and distribution of SSR-motifs was also predicted in the present study. EST-SSRs appear to be dispersed unevenly across the F. oxysproum f. sp. lycopersici genome, and there is a higher density of EST-SSRs on chromosome 1 . This observation were consistent with the observation of Singh et al. (2011 a), where they mentioned that the SSR repeat motif density in F. graminear- um genome was higher in chromosome 1 relative to other chromosomes.

Role of microsatellites in regulation of gene expression and in the evolution of gene regulation are well documented (Li et al., 2002, 2004). Polyleucine and polarginine repeats were reported as abundant amino acids in coding regions of $F$. oxysporum f. sp. lycopersici. In regulatory regions, changes in SSR motif length will necessary change the length of DNA in that region, thereby altering the local spatial relationship of transcription factor interactions (Kashi and King 2006).

Microsatellites, generally, show a decrease in abundance with increasing repeat length (Grover et al., 2007) and similar results were obtained in present study, where hexa-repeats were found least abundant in the genome. The longest hexanucleotide repeat motifs in $F$. oxysporum f. sp. oxysporum were found to be GGGTTA and similar repeat motif was reported in $P$. triticina and $P$ graminis $f$. sp. tritici (Singh et al., 2011 b). The rationale behind the categorization of SSR motifs on the basis of length of SSR tracts (Class I, II and III) is that longer perfect repeats are highly polymorphic as noticed in case of $F$. graminearum (Singh et al., 2011 a) and Fusarium oxysporum (Mahfooz et al., 2012). Microsatellites in Class III tended to be less variable, representing sites where SSR expansion may occasionally occur but its probability is limited due to a smaller chance of slipped-strand impairing over the shorter SSR template (Temnykh et al., 2001).

In conclusion, the present study has summarized information on cataloging SSRs along with their genomic and chromosomal positions, distribution and dynamics in the 
genome of $F$. oxysporum $\mathrm{f}$. sp. lycopersici. This information will be very useful for in-depth understanding of fungus evolutionary leading to the formation of repeats in the genome, diversity analysis, population genetics, race identification and acquisition of new virulence.

\section{Acknowledgements}

The authors gratefully acknowledge the financial assistance under project 'Outreach project on Phytophthora, Fusarium and Ralstonia disease in horticulture and field crops' from Indian Council of Agricultural Research (ICAR), India.

\section{References}

Balmas V, Scherm B, Di Primo P, Rau D, Marcello A, Migheli Q (2005). Molecular characterization of vegetative compatibility groups in Fusarium oxysporum f. sp. radicis-lycopersici and $\mathrm{f}$. sp. lycopersici by random amplification of polymorphic DNA and microsatellite-primed PCR. European J Plant Pathol 111:1-8.

Borrero C, Trillas MI, Ordales J, Tello JC, Aviles M (2004). Predictive factors for the suppression of Fusarium wilt of tomato in plant growth media. Phytopathology 94:1094-1101.

Breuillin F, Dutech C, Robin C (2006). Genetic diversity of the chestnut blight fungus Cryphonectria parasitica in four French populations assessed by microsatellite markers. Mycol Res 110:288-296.

Cai G, Gale LR, Schneider RW, Kistler HC, Davis RM, Elias KS, Miyao EM (2003). Origin of race 3 of Fusarium oxysporum f. sp. lycopersici at a single site in California. Phytopathology 93:1014-1022.

Elias KS, Schneider RW, Lear MM (1991). Analysis of vegetative compatibility groups in nonpathogenic populations of Fusarium oxysporum isolated from symptomless tomato roots. Can J Bot 69:2089-2094.

Grover A, Aishwarya V, Sharma PC (2007). Biased distribution of microsatellite motifs in the rice genome. Mol Genet Genomics 277:469-480.

Kashi Y, King DG (2006). Simple sequence repeats as advantageous mutators in evolution. Trends Genet 22(5):253-259.

Kim T-S, Booth JG, Gauch HG, Sun Q, Park J, Lee Y-H, Lee K (2008). Simple sequence repeats in Neurospora crassa: distribution, polymorphism and evolutionary inference. BMC Genomics 9:31.

Li Y-C, KorolAB, Fahima T, Nevo E (2004). Microsatellites within genes: Structure, function, and evolution. Mol Biol Evol 21(6):991-1007.

Li YC, Korol AB, Fahima T, Beiles A, Nevo E (2002). Microsatellites: genomic distribution, putative functions and mutational mechanisms: A review. Mol Ecol 11(12):2453.
Lievens B, van Baarlen P, Verreth C, van Kerckhove S, Rep M, Thomma BP (2009). Evolutionary relationships between Fusarium oxysporum f. sp. lycopersici and $F$. oxysporum $\mathrm{f}$. sp. radicis-lycopersici isolates inferred from mating type, elongation factor-1 alpha and exopolygalacturonase sequences. Mycol Res 113(10):1181-91.

Mahfooz S, Maurya DK, Srivastava AK, Kumar S, Arora DK (2012). A comparative in silico analysis on frequency and distribution of microsatellites in coding regions of three formae speciales of Fusarium oxysporum and development of EST-SSR markers for polymorphism studies. FEMS Microbiol Lett 328(1):54-60.

Martins WS, Lucas DCS, Neves KFS, Bertioli DJ (2009). WebSat - A web software for microsatellite marker development. Bioinformation 3(6):282-283.

Metzgar D, Bytof J, Wills C (2000). Selection against frame shift mutations limits microsatellite expansion in coding DNA. Genome Res 10:72-80.

Mishra KK, vr A, Pandey KK (2010). RAPD based genetic diversity among different isolates of Fusarium oxysporum f. sp. lycopersici and their comparative biocontrol. World J Microbiol Biotechnol 26(6):1079-1085.

Morgante M, Hanafey M, Powell W (2002). Microsatellites are preferentially associate with non-repetitive DNA in plant genomes. Nat Genet 30:194-200.

Richard G-F, Dujon B (1997). Trinucleotide repeats in yeast. Res Microbiol 148:731-744.

Rosewich UL, Pettway RE, Katan T, Kistler HC (1999). Population genetic analysis corroborates dispersal of Fusarium oxysporum f. sp. radicis-lycopersici from Florida to Europe. Phytopathology 89:623-630.

Singh R, Pandey B, Danishuddin M, Sheoran S, Sharma P, Chatrath R (2011b). Mining and survey of simple sequence repeats in wheat rust Puccinia sp. Bioinformation 7(6):291295.

Singh R, Sheoran S, Sharma P, Chatrath R(2011a). Analysis of simple sequence repeats (SSRs) dynamics in fungus Fusarium graminearum. Bioinformation 5(10):402-404.

Stallings RL, Ford AF, Nelson D, Torney DC, Hildebrand CE, Moyzis RK (1991). Evolution and distribution of (GT) $\mathrm{n}$ repetitive sequences in mammalian genomes. Genomics 10:807-815.

Temnykh S, DeClerck G, Lukashova A, Lipovich L, Cartinhour S, McCouch SR (2001). Computational and experimental analysis of microsatellites in rice (Oryza sativa L.): frequency, length variation, transposon associations, and genetic marker potential. Genome Res 11:1441-1452.

Tóth G, Gáspári Z, Jurka J (2000). Microsatellites in different eukaryotic genomes: survey and analysis. Genome Res 10:967-981. 